\title{
ON BRAIDED AND RIBBON UNITARY FUSION CATEGORIES
}

\author{
CÉSAR GALINDO
}

\begin{abstract}
We prove that every braiding over a unitary fusion category is unitary and every unitary braided fusion category admits a unique unitary ribbon structure.
\end{abstract}

\section{INTRODUCTION}

A unitary braided fusion category (UBFC) is a braided fusion category (BFC) over the complex numbers, where Hom-spaces have a Hilbert structure compatible with the tensor product and the braiding (see Subsection 2.1). The unitarity is the key additional structure for some applications of mathematics and theoretical physics. For example, in mathematics UBFCs give rise to unitary representations of the Artin braid group and finite depth subfactors of the hyperfinite $\mathrm{II}_{1}$ von Neumann factor [19]. For physics a unitary structure is important in order to construct unitary $(2+1)$-dimensional TQFT [16] 20] and algebraic models for exotic 2-dimensional physical (anyonic) systems [14 12. These last two applications make unitary modular categories the mathematical foundation for topological quantum computation [6] [11] 17. The above applications have renewed the interest in construction and study of properties of UBFCs.

Fusion categories are "quantum analogues" of finite groups, mainly because the prototypical example of a fusion category is $\operatorname{Rep}_{\mathbb{C}}(G)$, the category of finite dimensional complex representation of finite group $G$. It is also the case because in fusion categories there are phenomena such as nilpotency [8], solvability [4] and simplicity [5]. However, important differences exist between general fusion categories and $\operatorname{Rep}_{\mathbb{C}}(G)$, one of which is unitarity. The fusion category $\operatorname{Rep}_{\mathbb{C}}(G)$ admits a canonical unitary structure, but for example the Yang-Lee category does not [3]. In fact, there are families of premodular categories that do not admit unitary structures at all [15]. On the other hand, there are at least two infinite families of unitary premodular categories. One is associated with quantum groups [19] and the other one is related to finite groups [4]. The fusion categories of the latter family always admit a unique unitary structure [7].

Date: May 27, 2013.

2010 Mathematics Subject Classification. 20F36, 16W30, 18D10.

This work was partially supported by Vicerrectoría de Investigaciones de la Universidad de los Andes. 
In this note we are interested in the following natural questions. Does a BFC admit a unitary structure if the underlying fusion category is unitary? If it does, how many unitary ribbon structures does it admit? We prove that every braiding over a unitary fusion category is automatically unitary (see Theorem [3.2), and every UBFC admits a unique unitary ribbon structure (see Theorem 3.5).

Acknowlegements The author would like to thank Paul Bressler, Eric Rowell and Zhenghan Wang for useful comments.

\section{Preliminaries}

In this note, we will use basic theory of fusion categories and braided fusion categories. For further details on these topics, we refer the reader to [3]. In this section we recall some definitions and results on unitary fusion categories. Much of the material which appears here can be found in [16].

2.1. Unitary fusion categories. A $C^{*}$-category $\mathcal{D}$ is a $\mathbb{C}$-linear abelian category with an involutive antilinear contravariant endofunctor $*$ which is the identity on objects, the hom-spaces $\operatorname{Hom}_{\mathcal{D}}(X, Y)$ are Hilbert spaces and the norms satisfy

$$
\|f g\| \leq\|f\|\|g\|, \quad\left\|f^{*} f\right\|=\|f\|^{2},
$$

for all $f \in \operatorname{Hom}_{\mathcal{D}}(X, Y), g \in \operatorname{Hom}_{\mathcal{D}}(Y, Z)$, where $f^{*}$ denote the image of $f$ under $*$.

Let $X$ and $Y$ be objects in a $C^{*}$-category. A morphism $u: X \rightarrow Y$ is unitary if $u u^{*}=\operatorname{id}_{Y}$ and $u^{*} u=\operatorname{id}_{X}$. A morphism $a: X \rightarrow X$ is self-adjoint if $a^{*}=a$.

Remark 2.1. Every isomorphism in a $C^{*}$-category has a polar decomposition, i.e., if $f: X \rightarrow Y$ is an isomorphism, then $f=u a$ where $a: X \rightarrow X$ is self-adjoint and $u: X \rightarrow Y$ is unitary, see [1, Proposition 8].

A unitary fusion category is a fusion category $\mathcal{C}$, where $\mathcal{C}$ is a $C^{*}$ category, the constraints are unitary and $(f \otimes g)^{*}=f^{*} \otimes g^{*}$, for every pair of morphisms $f, g$ in $\mathcal{C}$.

Remark 2.2.

(1) A unitary fusion category is a fusion category with an additional structure. Hence, a fusion category could have more than one unitary structure. All examples known to the author admit a unique unitary structure. Moreover, in [7, Theorem 5.20] it was proved that every weakly group-theoretical fusion category admits a unique unitary structure.

(2) If $\mathcal{C}$ is a unitary fusion category, we can find bases such that the $F$ matrices $\left(F_{l}^{i j k}\right)_{n, m}=F_{l ; n, m}^{i, j, k}$ are unitary, where $\left\{F_{l ; n, m}^{i, j, k}\right\}$ are the $6 j$ symbols (see [17] or [16] for the definition of $6 j$-symbols). Conversely,

\footnotetext{
${ }^{1}$ This answer Problem 3.3 in http://aimpl.org/fusioncat/, posted by Zhenghan Wang.
} 
if for a fusion category $\mathcal{C}$ it is possible to find basis such that the $F$-matrices $\left(F_{l}^{i j k}\right)_{n, m}=F_{l ; n, m}^{i, j, k}$ are unitary, then $\mathcal{C}$ is a unitary fusion category. See [18, Section 4].

\section{BRAIDING AND MODUlar STRUCTURES OVER UNITARY FUSION CATEGORIES ARE UNITARY}

3.1. The center of a unitary fusion category. We shall recall the definition of the center $\mathcal{Z}(\mathcal{C})$ of a monoidal category $\mathcal{C}$, see [9, Chapter XIII]. The objects of $\mathcal{Z}(\mathcal{C})$ are pairs $\left(Y, c_{-, Y}\right)$, where $Y \in \mathcal{C}$ and $c_{X, Y}: X \otimes Y \rightarrow Y \otimes X$ are isomorphisms natural in $X$ satisfying $c_{X \otimes Y, Z}=\left(c_{X, Z} \otimes \mathrm{id}_{Y}\right)\left(\operatorname{id}_{X} \otimes c_{Y, Z}\right)$ and $c_{I, Y}=\operatorname{id}_{Y}$, for all $X, Y, Z \in \mathcal{C}$. A morphism $f:\left(X, c_{-, X}\right) \rightarrow\left(X, c_{-, X}\right)$ is a morphism $f: X \rightarrow Y$ in $\mathcal{C}$ such that $\left(f \otimes \operatorname{id}_{W}\right) c_{W, X}=c_{W, Y}\left(\operatorname{id}_{W} \otimes f\right)$ for all $W \in \mathcal{C}$.

The center is a braided monoidal category with structure given as follows:

- the tensor product is $\left(Y, c_{-, Y}\right) \otimes\left(Z, c_{-, Z}\right)=\left(Y \otimes Z, c_{-, Y \otimes Z}\right)$, where

$c_{X, Y \otimes Z}=\left(\operatorname{id}_{Y} \otimes c_{X, Z}\right)\left(c_{X, Y} \otimes \operatorname{id}_{Z}\right): X \otimes Y \otimes Z \rightarrow Y \otimes Z \otimes X$,

for all $X \in \mathcal{C}$,

- the identity element is $\left(I, c_{-, I}\right), c_{Z, I}=\mathrm{id}_{Z}$

- the braiding is given by the morphism $c_{X, Y}$.

If $\mathcal{C}$ is a unitary fusion category the unitary center $\mathcal{Z}^{*}(\mathcal{C})$ is defined as the full tensor subcategory of $\mathcal{Z}(\mathcal{C})$, where $\left(X, c_{-, X}\right) \in \mathcal{Z}^{*}(\mathcal{C})$ if and only if $c_{W, X}: W \otimes X \rightarrow X \otimes W$ is unitary for all $W \in \mathcal{C}$.

Proposition 3.1. Let $\mathcal{C}$ be a unitary fusion category then $\mathcal{Z}^{*}(\mathcal{C})=\mathcal{Z}(\mathcal{C})$.

Proof. Let $\left(X, c_{-, X}\right)$ be an object in $\mathcal{Z}(\mathcal{C})$. By [7, Proposition 5.24.] or [13, Theorem 6.4], the inclusion functor $\mathcal{Z}^{*}(\mathcal{C}) \subseteq \mathcal{Z}(\mathcal{C})$ is a tensor equivalence. Therefore, there is an object $\left(Y, c_{-, Y}\right)$ in $\mathcal{Z}^{*}(\mathcal{C})$ and an isomorphism $f$ : $\left(X, c_{-, X}\right) \rightarrow\left(Y, c_{-, Y}\right)$ in $\mathcal{Z}(\mathcal{C})$. By Remark 2.1 there exists a unitary arrow $u:\left(X, c_{-, X}\right) \rightarrow\left(Y, c_{-, Y}\right)$. Hence, for every $W \in \mathcal{C}$,

$$
c_{W, X}=\left(u \otimes \mathrm{id}_{W}\right)^{*} \circ c_{W, Y}\left(\mathrm{id}_{W} \otimes u\right),
$$

so $c_{W, X}$ is a unitary arrow and $\left(X, c_{-, X}\right) \in \mathcal{Z}^{*}(\mathcal{C})$.

A braiding over a unitary fusion category $\mathcal{C}$ is called unitary braiding if the morphism $c_{X, Y}$ is unitary for any pair of objects $X, Y \in \mathcal{C}$.

Theorem 3.2. Every braiding of a unitary fusion category is unitary.

Proof. Let $\mathcal{C}$ be a unitary fusion category and let $c$ be a braiding. It is easy to see that the braiding $c$ defines an inclusion functor $\mathcal{C} \hookrightarrow \mathcal{Z}(\mathcal{C}), X \mapsto$ $\left(X, c_{X,-}\right)$. Proposition 3.1 implies that $c_{X, W}$ is unitary for every $W \in \mathcal{C}$. Remark 3.3.

(1) Theorem 3.2 implies that if the $F$-matrices $\left(F_{l}^{i j k}\right)_{n, m}=F_{l ; n, m}^{i, j, k}$ are unitary, then the $R$-matrices of the braiding are always unitarily diagonalizable. 
(2) A Kac algebra $(H, m, \Delta, *)$ is a semisimple Hopf algebra such that $(H, *)$ is a $C^{*}$-algebra and the maps $\Delta$ and $\varepsilon$ are $C^{*}$-algebra maps. Theorem 3.2 implies that every $R$-matrix in a Kac algebra is unitary in the sense that $R^{*}=R^{-1}$.

3.2. Ribbon structures on unitary fusion categories. If $\mathcal{C}$ is a fusion category, then for every $f \in \operatorname{Hom}_{\mathcal{C}}(X, Y)$ the transpose of $f$, is defined by

$$
{ }^{t} f:=\left(\operatorname{id}_{X^{*}} \otimes \operatorname{ev}_{Y}\right)\left(\operatorname{id}_{X^{*}} \otimes f \otimes \operatorname{id}_{Y^{*}}\right)\left(\operatorname{coev}_{X} \otimes \operatorname{id}_{Y^{*}}\right) \in \operatorname{Hom}_{\mathcal{C}}\left(Y^{*}, X^{*}\right) .
$$

A twist on a braided fusion category $\mathcal{C}$ is a natural automorphism of the identity functor $\theta \in \operatorname{Aut}\left(\operatorname{Id}_{\mathcal{C}}\right)$, such that

$$
\theta_{X \otimes Y}=\left(\theta_{X} \otimes \theta_{Y}\right) c_{Y, X} c_{X, Y}
$$

for all $X, Y \in \mathcal{C}$. A twist is called a ribbon structure if ${ }^{t} \theta_{X}=\theta_{X^{*}}$. A fusion category with a ribbon structure is called a ribbon fusion category. Each ribbon structure $\theta$ defines a quantum dimension function by $\operatorname{dim}_{\theta}(X)=\operatorname{ev}_{X} c_{X, X^{*}}\left(\theta_{X} \otimes \operatorname{id}_{X^{*}}\right) \operatorname{coev}_{X}$.

We shall denote by $\mathrm{Aut}_{\otimes}\left(\operatorname{Id}_{\mathcal{C}}\right)_{(+,-)}$the abelian group of tensor automorphisms $\gamma$ of the identity such that $\gamma_{X}= \pm \mathrm{id} X$ for every simple object $X \in \mathcal{C}$.

Proposition 3.4. Let $\mathcal{C}$ be a braided fusion category. If the set of ribbon structures is not empty, it is a torsor under Aut $\otimes\left(\operatorname{Id}_{\mathcal{C}}\right)_{(+,-)}$.

Proof. Let $\theta$ and $\theta^{\prime}$ be ribbon structures. It is easy to see that $\gamma:=\theta^{-1} \theta^{\prime}$ : $\operatorname{Id}_{\mathcal{C}} \rightarrow \operatorname{Id}_{\mathcal{C}}$ is a tensor automorphism of the identity. For every simple object, we have $\theta_{X}=\theta(X)$ id $_{X}, \theta_{X}^{\prime}=\theta(X)^{\prime} \mathrm{id}_{X}, \gamma_{X}=\gamma(X)$ id $_{X}$ for some $\gamma(X), \theta(X), \theta(X)^{\prime} \in \mathbb{C}^{*}$ and $\theta(X)^{\prime}=\gamma(X) \theta(X)$. Since $\theta^{\prime}$ is a ribbon structure, for every simple object $X \in \mathcal{C}$, $\operatorname{dim}_{\theta^{\prime}}(X)=\operatorname{dim}_{\theta^{\prime}}\left(X^{*}\right)$. On the other hand, $\operatorname{dim}_{\theta^{\prime}}(X)=\gamma(X) \operatorname{dim}_{\theta}(X)$. Therefore $\gamma(X)=\gamma\left(X^{*}\right)$ and, since $\gamma\left(X^{*}\right)=\gamma(X)^{-1}$ we conclude that $\gamma$ has order two.

Conversely, if $\gamma$ is an automorphism of the identity such that $\gamma_{X}= \pm \mathrm{id}_{X}$ for every simple object, then, for every ribbon structure $\theta$, the natural isomorphism $\theta^{\prime}=\theta \gamma$ is a new ribbon structure.

If $\mathcal{C}$ is a unitary fusion category a ribbon structure on $\mathcal{C}$ is called unitary ribbon structure if $\theta_{X}$ is unitary, $\left(\operatorname{coev}_{X}\right)^{*}=\operatorname{ev}_{X}\left(c_{X, X^{*}}\right)\left(\theta_{X} \otimes \mathrm{id}_{X^{*}}\right)$ and $\left(\operatorname{ev}_{X}\right)^{*}=\left(\operatorname{id}_{X^{*}} \otimes \theta_{X}^{-1}\right)\left(c_{X^{*}, X}\right)^{-1} \operatorname{coev}_{X}$ for all $X \in \mathcal{C}$. A unitary fusion category with a unitary ribbon structure is called a unitary ribbon fusion category or unitary premodular category. In a unitary ribbon fusion category

$$
\operatorname{dim}_{\theta}(X)=\operatorname{ev}_{X}\left(c_{X, X^{*}}\right)\left(\theta_{X} \otimes \mathrm{id}_{X^{*}}\right) \operatorname{coev}_{X}=\left(\operatorname{coev}_{X}\right)^{*} \operatorname{coev}_{X},
$$

therefore, the quantum dimension of every object is a positive number.

Theorem 3.5. Every braided fusion category with a unitary structure admits a unique unitary ribbon structure. 
Proof. By [10, Proposition 2.4] every braided unitary fusion category admits a canonical unitary ribbon structure. Let $\theta_{c}$ the canonical ribbon structure associated to $c$. By Proposition 3.4, if $\theta^{\prime}$ is another unitary ribbon structure, then there is $\gamma \in \operatorname{Aut}{ }_{\otimes}\left(\operatorname{Id}_{\mathcal{C}}\right)_{(+,-)}$such that $\theta^{\prime}=\theta_{c} \gamma$. If $\gamma$ is not the identity there is a simple object $X \in \mathcal{C}$ such that $\gamma_{X}=-\mathrm{id} X$, then $\operatorname{dim}_{\theta^{\prime}}(X)=$ $-\operatorname{dim}_{\theta_{c}}(X)<0$, but the quantum dimension of every object of any unitary ribbon structure is positive. Therefore $\gamma$ is the identity and $\theta_{c}$ is unique.

Remark 3.6. It follows from Theorem 3.5 that if a unitary braided fusion category is non-degenerate (see [2] for a definition), then it admits a unique unitary modular structure.

\section{REFERENCES}

1. J. Baez, "Higher-Dimensional Algebra II. 2-Hilbert Spaces." Adv. Math. 127 (1997), 125-189.

2. V. Drinfeld, S. Gelaki, D. Nikshych, V. Ostrik, " On Braided Fusion Categories I." Selecta Math. N.S. 16, 1 (2010) 1-119.

3. P. Etingof, D. Nikshych, V. Ostrik, "On fusion categories." Ann. of Math. (2) 162 (2005), no. 2, 581-642.

4. P. Etingof, D. Nikshych, V. Ostrik, "Weakly group-theoretical and solvable fusion categories." Adv. Math. 226 (2011) 176205.

5. P. Etingof, D. Nikshych, V. Ostrik, "Fusion categories and homotopy theory". Quantum Topology 1, no. 3 (2010): 209-273. Preprint arXiv:0909.3140.

6. M. Freedman, "P/NP, and the quantum field computer." Proc. Natl. Acad. Sci. 95 (1998), no. 1, 98-101.

7. C. Galindo, S.-M. Hong, E. Rowell, "Generalized and quasi-localizations of braid group representations", Int. Math. Res. Not., Vol. 2013, No. 3, pp. 693-731.

8. S. Gelaki, D. Nikshych, "Nilpotent fusion categories", Adv. Math., 217 (2008), 1053-1071.

9. C. Kassel, Quantum Groups, Graduate Texts in Mathematics 155, SpringerVerlag, New York, 1995.

10. M. Mueger, "Galois theory for braided tensor categories and the modular closure." Adv. Math. 150 (2000), 151-201.

11. A. Kitaev, "Fault-tolerant quantum computation by anyons." Ann. Physics 303,1 (2003), 2-30.

12. G. Moore, N. Read," "Nonabelions in the fractional quantum Hall efect." Nuclear Physics B, Volume 360, 362-396..

13. M. Mueger, "From subfactors to categories and topology II. The quantum double of tensor categories and subfactors." J. Pure Appl. Alg. 180 (2003), 159-219.

14. N. Read, E. Rezayi, "Beyond paired quantum Hall states: Parafermions and incompressible states in the first excited Landau level." Phys. Rev. B 59 (1999), 8084.

15. E. Rowell, "On a family of non-unitarizable ribbon categories." Math. Z. 250 (2005), no. 4, 745-774

16. V. G. Turaev, Quantum Invariants of Knots and 3-Manifolds., Revised Edition, de Gruyter Studies in Mathematics 18, Walter de Gruyter \& Co., Berlin, 2010.

17. Z. Wang, Topological quantum computation., CBMS Regional Conference Series in Mathematics, 112, 2010.

18. S. Yamagami, "Polygonal presentations of semisimple tensor categories.", J. Math. Soc. Japan, 54, (2002), 61-88. 
19. H. Wenzl, " $C^{*}$-tensor categories from quantum groups", J. Amer. Math. Soc., 11, (1998), 261-282.

20. E. Witten, "Topological quantum field theory.", Comm. Math. Phys., 117, (1998), 353-386.

Departamento de Matemáticas, Universidad de los Andes, Bogotá, Colombia

E-mail address: cn.galindo1116@uniandes.edu.co, cesarneyit@gmail.com 